\title{
Optimal Research and Numerical Simulation for Scheduling No-Wait Flow Shop in Steel Production
}

\author{
Huawei Yuan, ${ }^{1}$ Yuanwei Jing, ${ }^{1}$ Jinping Huang, ${ }^{2}$ and Tao $\operatorname{Ren}^{3}$ \\ ${ }^{1}$ School of Information Science and Engineering, Northeastern University, Shenyang 110819, China \\ ${ }^{2}$ Shenyang Control Center of Petro-China Pipeline Company, Shenyang 110031, China \\ ${ }^{3}$ Software College, Northeastern University, Shenyang 110819, China \\ Correspondence should be addressed to Tao Ren; chinarentao@163.com
}

Received 8 October 2013; Revised 14 December 2013; Accepted 20 December 2013

Academic Editor: Yuri Sotskov

Copyright (C) 2013 Huawei Yuan et al. This is an open access article distributed under the Creative Commons Attribution License, which permits unrestricted use, distribution, and reproduction in any medium, provided the original work is properly cited.

\begin{abstract}
This paper considers the $m$-machine flow shop scheduling problem with the no-wait constraint to minimize total completion time which is the typical model in steel production. First, the asymptotic optimality of the Shortest Processing Time (SPT) first rule is proven for this problem. To further evaluate the performance of the algorithm, a new lower bound with performance guarantee is designed. At the end of the paper, numerical simulations show the effectiveness of the proposed algorithm and lower bound.
\end{abstract}

\section{Introduction}

Steel-making is a multistage process in which melted iron is converted into steel products sequentially by the processes of converting furnace, heating furnace, and rolling mill. Distinctly, this is a very typical model of the flow shop. Differently, in the steel production, the hot work-in-processes can not wait between two successive operations. For example, a slab has to reach a rolling temperature through the heat furnace before it can be processed by the rolling mill. If a heated slab waits for a long time in front of the machine, its temperature will drop significantly. Once the temperature of a slab falls below the rolling temperature, the reheating must be executed, which will consume a lot of energy. Furthermore, the size of the work-in-process in steel-making is especially large, which limits the storage capacity of the buffer between two successive machines. As minimizing the criterion of total completion time (TCT; the detail about TCT objective can be found in $[1,2]$ ) can effectively reduce the in-process inventory, the research of no-wait flow shop with TCT objective is reasonably effective for iron and steel industry.

With the standard scheduling notation suggested by Graham et al. in 1979 [3], the no-wait flow shop scheduling problem to minimize TCT can be denoted by $F m \mid$ no-wait $\mid \Sigma C_{j}$.
Röck [4] indicated the strong NP-hardness for problem $F 2 \mid$ no-wait $\mid \Sigma C_{j}$ which means that the optimal solution for the general problem, $F m \mid$ no-wait $\mid \Sigma C_{j}$, cannot be obtained in polynomial time unless $\mathrm{P}=\mathrm{NP}$. Allahverdi and Aldowaisan [5] considered the $F 2 \mid$ no-wait, $s_{j k} \mid \Sigma C_{j}$ problem, where $s_{j k}$ denotes that the setup time is sequence dependent. Optimal solutions were obtained for two special flow shops and a dominance relation is developed for the general problem. Several heuristic algorithms with polynomial computational time are constructed. Allahverdi and Aldowaisan [6] addressed problem $F 3 \mid$ no-wait, $s_{j} \mid \Sigma C_{j}$, where $s_{j}$ denotes that the setup times are separate from processing times and sequence independent. Optimal solutions and a dominance relation were presented, respectively, for certain cases and the general case. Five heuristic algorithms were developed and evaluated for small and large number of jobs. Aldowaisan and Allahverdi [7] provided new heuristics and compared the performance of these proposed algorithms with that of three existing heuristics for problem $F m \mid$ no-wait $\mid \Sigma C_{j}$. Gao et al. [8] present two constructive heuristics, improved standard deviation heuristic (ISDH), and improved Bertolissi heuristic (IBH) for problem $F m \mid$ no-wait $\mid \Sigma C_{j}$, and propose four composite heuristics, using the insertion-based local search method and iteration operator to improve the solutions of the ISDH and IBH. Allahverdi and Aydilek [9] discussed problem 
$F m \mid$ no-wait $\mid \Sigma C_{j}, C_{\max } \leq \theta$, where $\theta$ is a certain value. An algorithm is proposed to find an upper bound on the makespan in case the upper bound is not given or unknown. Given the upper bound on makespan, a proposed algorithm and a genetic algorithm are utilized for solving the problem. A survey of problem $F m \mid$ no-wait $\mid \Sigma C_{j}$ can be found in [10].

In this paper, the asymptotic optimality of the Shortest Processing Time (SPT) first SPT rule is proved for problem $F m \mid$ no-wait $\mid \Sigma C_{j}$ in the sense of probability limit, where the research is restricted to the permutation schedule (the details about permutation schedule can be found in $[1,2])$. To further evaluate the strategy, a new lower bound with performance guarantee is designed. At the end of the paper, numerical simulations show the convergence of the algorithm and the effectiveness of the lower bound.

The remainder of the paper is organized as follows. The problem is formulated in Section 2, the asymptotic optimality of the SPT rule is proved in Section 3. The new lower bound and computational experiments are presented in Sections 4 and 5, respectively. And in Section 6, this paper is closed by the conclusions.

\section{Problem Specification}

In no-wait flow shop problem, a set of $n$ jobs has to be sequentially processed on $m$ different machines without preemption. Each job $j, j=1,2, \ldots, n$, passes through the $m$ machines in identical order and requires processing time $p(i, j)$ on machine $i, i=1,2, \ldots, m$. It is assumed that the processing times are independently and identically distributed (i.i.d.) random variables, defined on the interval $(0,1]$. At any given time each machine can handle at most one job and each job can be processed on at most one machine. Jobs are available simultaneously, and the permutation schedule is considered; that is, all jobs are processed on all machines in the same order. The jobs cannot wait between two successive machines and the intermediate storage is zero. A job that was finished on a given machine must remain on it until the next machine completes the processing of the preceding job. Let $D(i, j)$ denote the time that job $j, j=1,2, \ldots, n$, actually takes to depart machine $i, i=1,2, \ldots, m$. The time job $j$ starts its processing at the first machine is denoted by $D(0, j)$. The completion time of job $j$ is denoted by $D_{j}$. The objective is to find a sequence of jobs under the constraint of permutation schedule to minimize the sum of completion times on the final machine; that is, $\min \sum_{j=1}^{n} D_{j}$.

\section{Proof of Asymptotic Optimality for SPT Rule}

The SPT rule is actually a heuristic in which the jobs are scheduled in nondecreasing order according to value $P_{j}=$ $\sum_{i=1}^{m} p(i, j)$. With the tool of asymptotic analysis, the asymptotic optimality of the SPT rule for problem $F m \mid$ no-wait $\mid \Sigma C_{j}$ is introduced as follows.

Theorem 1. Let the processing times $p(i, j), j=1,2, \ldots, n, i=$ $1,2, \ldots, m$, be independent random variables having the same continuous distribution with bounded density $\varphi(\cdot)$ defined on $(0,1]$. Then, with probability one

$$
\lim _{n \rightarrow \infty} \frac{Z^{S P T}}{n^{2}}=\lim _{n \rightarrow \infty} \frac{Z^{*}}{n^{2}},
$$

where $Z^{S P T}$ is the objective value obtained by the SPT rule, and $Z^{*}$ is the optimal value.

Proof. The jobs to be scheduled are index in the SPT sequence. For arbitrary job number $j, j=1,2, \ldots, n$, the processing times that appear in a SPT sequence can be expressed as follows

$$
\begin{array}{ccccccc}
p(1,1) & p(1,2) & \ldots & p(1, j) & & & \\
p(2,1) & p(2,2) & \cdots & p(2, j) & & & \\
& p(3,1) & p(3,2) & \cdots & p(3, j) & & \\
& & \ddots & & & \ddots & \\
& & & p(m, 1) & p(m, 2) & \cdots & p(m, j) .
\end{array}
$$

We add the processing times of job 1 at the left bottom and that of job $j$ at the right top, respectively, and obtain a matrix $A$ as follows:

$$
A=\left(\begin{array}{cccccccc}
p(1,1) & p(1,2) & \cdots & p(1, j) & p(2, j) & p(3, j) & \cdots & p(m, j) \\
p(1,1) & p(2,1) & p(2,2) & \cdots & p(2, j) & p(3, j) & \cdots & p(m, j) \\
p(1,1) & p(2,1) & p(3,1) & p(3,2) & \cdots & p(3, j) & \cdots & p(m, j) \\
\vdots & \vdots & \vdots & \ddots & & & \ddots & \vdots \\
p(1,1) & p(2,1) & p(3,1) & \cdots & p(m, 1) & p(m, 2) & \cdots & p(m, j)
\end{array}\right) .
$$


For a given SPT sequence, with matrix $A$, we have

$$
\begin{aligned}
\sum_{k=1}^{j} & \sum_{i=1}^{m} p(i, k) \\
& <\sum_{i=1}^{m}\left(\sum_{i=1}^{i-1} p(i, 1)+\sum_{k=1}^{j} p(i, k)+\sum_{i=i+1}^{m} p(i, j)\right) .
\end{aligned}
$$

Summing all over the jobs and dividing $m$ on both sides of (4), we have

$$
\begin{aligned}
Z^{(1)} 1 & =\frac{1}{m} \sum_{j=1}^{n} \sum_{k=1}^{j} \sum_{i=1}^{m} p(i, k) \\
& <\frac{1}{m} \sum_{j=1}^{n} \sum_{i=1}^{m}\left(\sum_{i=1}^{i-1} p(i, 1)+\sum_{k=1}^{j} p(i, k)+\sum_{i=i+1}^{m} p(i, j)\right) \\
& =Z^{(2)}
\end{aligned}
$$

where $Z^{(1)}$ and $Z^{(2)}$ are the lower bound values of $\mathrm{LB}(1)$ and $\mathrm{LB}(2)$, respectively. Denote the completion time of job $j$ in $\mathrm{LB}(1), \mathrm{LB}(2)$, and the SPT sequence by $D_{j}^{(1)}, D_{j}^{(2)}$, and $D_{j}^{\mathrm{SPT}}$, respectively. For the $n$ jobs, the gap between the SPT sequence and the lower bound is

$$
\begin{aligned}
& I_{n}= D_{n}^{\mathrm{SPT}}-D_{n}^{(2)}<C_{n}^{\mathrm{SPT}}-C_{n}^{(1)} \\
&= \max _{1 \leq\left|J_{1}\right|,\left|J_{2}\right|, \ldots,\left|J_{m}\right| \leq n}\left\{\sum_{j \in J_{1}} p(1, j)\right. \\
&\left.+\sum_{j \in J_{2}} p(2, j)+\cdots+\sum_{j \in J_{m}} p(m, j)\right\} \\
&-\frac{1}{m} \sum_{i=1}^{m}\left(\sum_{i=1}^{i-1} p(i, 1)+\sum_{k=1}^{j} p(i, k)+\sum_{i=i+1}^{m} p(i, j)\right) \\
&=\left\{\sum_{1 \leq\left|J_{1}\right|,\left|J_{2}\right|, \ldots,\left|J_{m}\right| \leq n} p(1, j)+\sum_{j \in J_{2}} p(2, j)\right. \\
&\left.+\cdots+\sum_{j \in J_{m}} p(m, j)\right\} \\
&\left.-\sum_{i=1}^{m}\left(\sum_{i=1}^{i-1} p(i, 1)+\sum_{k=1}^{j} p(i, k)+\sum_{i=i+1}^{m} p(i, j)\right)\right)
\end{aligned}
$$

where $J_{i}$ denotes the set that includes the jobs on machine $i$, $i=1,2, \ldots, m$, in the critical path. Dividing $n$ on both sides of (6) and taking limit, we have

$$
\begin{aligned}
& \lim _{n \rightarrow \infty} \frac{I_{n}}{n} \\
& =\lim _{n \rightarrow \infty} \frac{D_{n}^{\mathrm{SPT}}}{n}-\lim _{n \rightarrow \infty} \frac{D_{n}^{(2)}}{n} \\
& <\lim _{n \rightarrow \infty} \frac{D_{n}^{\mathrm{SPT}}}{n}-\lim _{n \rightarrow \infty} \frac{D_{n}^{(1)}}{n} \\
& =\frac{1}{m} \\
& \times\left(\lim _{n \rightarrow \infty} \frac{1}{n}\right. \\
& \times \max _{1 \leq\left|J_{1}\right|,\left|J_{2}\right|, \ldots,\left|J_{m}\right| \leq n}\left\{\sum_{j \in J_{1}} p(1, j)\right. \\
& \left.+\sum_{j \in J_{2}} p(2, j)+\cdots+\sum_{j \in J_{m}} p(m, j)\right\} \\
& -\lim _{n \rightarrow \infty} \frac{1}{n} \sum_{i=1}^{m} \sum_{k=1}^{j} p(i, k) \\
& \left.-\lim _{n \rightarrow \infty} \frac{1}{n} \sum_{i=1}^{m}\left(\sum_{i=1}^{i-1} p(i, 1)+\sum_{i=i+1}^{m} p(i, j)\right)\right) \\
& =\frac{1}{m}\left(E\left(p_{j}\right)-E\left(p_{j}\right)\right)-\frac{1}{m} \times 0=0,
\end{aligned}
$$

where $E\left(p_{j}\right)$ denotes the expectation of the processing times, and the penultimate inequality of (7) is because of the Law of Large Numbers. Let $I_{\max }$ be the maximum value of $I_{n}$ among the $n$ jobs. Therefore, we have

$$
\begin{gathered}
\lim _{n \rightarrow \infty} \frac{I_{\max }}{n}=0, \\
\lim _{n \rightarrow \infty} \frac{D_{n}^{(1)}}{n}=\lim _{n \rightarrow \infty} \frac{D_{n}^{(2)}}{n}=\lim _{n \rightarrow \infty} \frac{D_{n}^{\mathrm{SPT}}}{n} .
\end{gathered}
$$

Summing all over the $n$ jobs, we have

$$
Z^{(1)}+n I_{\max } \leq Z^{*} \leq Z^{\mathrm{SPT}} \leq Z^{(2)}+n I_{\max }
$$

Dividing $n^{2}$ on both sides of (8) and taking limit, we have

$$
\begin{aligned}
& \lim _{n \rightarrow \infty} \frac{Z^{(1)}}{n^{2}}+\lim _{n \rightarrow \infty} \frac{n I_{\max }}{n} \\
& \quad \leq \lim _{n \rightarrow \infty} \frac{Z^{*}}{n^{2}} \leq \lim _{n \rightarrow \infty} \frac{Z^{\mathrm{SPT}}}{n^{2}} \leq \lim _{n \rightarrow \infty} \frac{Z^{(2)}}{n^{2}}+\lim _{n \rightarrow \infty} \frac{n I_{\max }}{n^{2}} .
\end{aligned}
$$

With limits (8) and (9), we obtain the result of the theorem. 


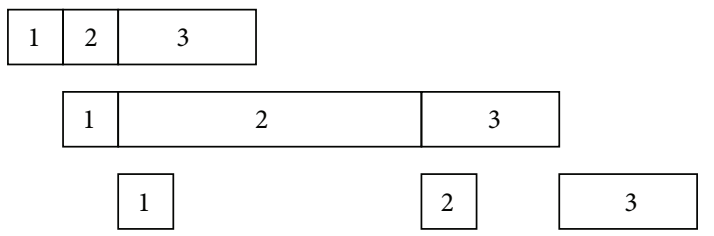

FIGURE 1: Gantt chart of Example 1.

\section{The New Lower Bound}

As the stronger NP-hardness of the problem, the lower bound is usually a substitution of the optimal solution. In 2013, Bai and Ren [11] presented an asymptotically optimal lower bound, LB1, for problem $F m \mid$ no-wait $\mid \Sigma C_{j}$ as follows:

$$
\mathrm{LB1}=\max \{X 1, X 2, X 3\},
$$

where

$$
\begin{gathered}
X 1=\frac{1}{m} \sum_{j=1}^{n}\left(\sum_{k=1}^{j} \sum_{i=1}^{m} p(i, k)+\sum_{i=1}^{m}(m-i) p(i, 1)\right. \\
\left.+\sum_{i=1}^{m}(i-1) p(i, j)\right) \\
X 2=\sum_{j=1}^{n}\left(\sum_{k=1}^{j} p(1, k)+\sum_{i=2}^{m} p(i, j)\right) \\
X 3=\sum_{j=1}^{n}\left(\sum_{i=1}^{m-1} p(i, 1)+\sum_{k=1}^{j} p(m, k)\right) .
\end{gathered}
$$

This lower bound can deal with problem $F m \mid$ no-wait $\mid \Sigma C_{j}$ without any change. But in some cases, LB1 does not work well. For instance, consider the following example (see Figure 1).

Example 1. There is three-machine flow shop problem with three jobs. The processing times of these jobs are $p(1,1)=$ $p(2,1)=p(3,1)=1 ; p(1,2)=p(3,2)=1, p(2,2)=10$; and $p(1,3)=p(2,3)=p(3,3)=5$. Therefore, the optimal sequence is $\{1,2,3\}$ and the optimal value is 38 (see Figure 1 ). For the associated LB1 value, we have

$$
\mathrm{LB1}=\max \{27,10,10\}=27 .
$$

And the gap between the optimal value and LB1 is $(38-27) / 27$ $\times 100 \% \approx 40.74 \%$. Obviously, the error is considerable. To improve the performance of LB1, a new lower bound, $\mathrm{LB}(3)$, is provided. Consider

$$
Z^{(3)}=\sum_{j=1}^{n} \max _{1 \leq i \leq m}\left\{\sum_{i^{\prime}=1}^{i-1} p\left(i^{\prime}, 1\right)+\sum_{j=1}^{n} p(i, j)+\sum_{i^{\prime \prime}=i+1}^{m} p\left(i^{\prime \prime}, n\right)\right\},
$$

where $Z^{(3)}$ is the lower bound value of $\mathrm{LB}(3)$. Calculate Example 1 with $\mathrm{LB}(3)$, and obtain the value 38 .
Theorem 2. For any instance of problem Fm $\mid$ no-wait $\mid \Sigma C_{j}$, we have

$$
Z^{1} \leq Z^{(3)}
$$

where $Z^{1}$ is the objective value of $L B 1$.

Proof. Consider a given optimal schedule for $F m \mid$ nowait $\mid \Sigma C_{j}$ in which the jobs are indexed from 1 to $n$. Denoting the completion time of job $j, 1 \leq j \leq n$, in $\operatorname{LB}(3)$ as $D_{j}^{(3)}$, we have

$$
D_{j}^{(3)}=\max _{1 \leq i \leq m}\left\{\sum_{i^{\prime}=1}^{i-1} p\left(i^{\prime}, 1\right)+\sum_{k=1}^{j} p(i, k)+\sum_{i^{\prime \prime}=i+1}^{m} p\left(i^{\prime \prime}, j\right)\right\} .
$$

Therefore, we have

$$
\begin{gathered}
D_{j}^{(3)} \geq \sum_{k=1}^{j} p(1, k)+\sum_{i=2}^{m} p(i, j), \\
D_{j}^{(3)} \geq \sum_{i=1}^{m-1} p(i, 1)+\sum_{k=1}^{j} p(m, k), \\
D_{j}^{(3)} \geq \frac{1}{m} \sum_{k=1}^{j} \sum_{i=1}^{m} p(i, k) \\
+\sum_{i=1}^{m}(m-i) p(i, 1)+\sum_{i=1}^{m}(i-1) p(i, j) .
\end{gathered}
$$

Summing all over the $n$ jobs, we can obtain the result of the theorem.

\section{Computational Results}

In this section, we designed a series of computational experiments to reveal the convergence of the SPT rule and the effectiveness of the new lower bound in different size problems. Firstly, we compare the SPT rule with LB(3) to show convergence trend. And then, we compare $\mathrm{LB}(3)$ with $\operatorname{LB}(2)$ to show the effectiveness of $\operatorname{LB}(3)$. Different combinations of jobs and machines are tests to show the performance variation when parameters vary. Combinations with five, ten, and 15 machines with 100, 200, 500, 1000, and 1500 jobs are tested for testing the SPT rule and lower bound $\mathrm{LB}(3)$. The processing times were randomly generated from a discrete uniform distribution on $[1,100]$, and a normal distribution with mean $(1+100) / 2$ and variance 49 , respectively. Ten different random tests for each combination of the parameters were performed, and the averages are reported.

The ratios $\mathrm{SPT} / \mathrm{LB}(3)$ showed in Table 1 are the objective values of the SPT rule to those of $\mathrm{LB}(3)$. From the data in the table, we can see that the ratios of SPT/LB(3) approach one as the number of jobs increases from 100 to 1500 with the fixed number of machines. For example, in 15 machines with uniform distribution, the ratio of $\mathrm{SPT} / \mathrm{LB}(3)$ drops 
TABLE 1: Results of SPT/LB(3).

\begin{tabular}{ccccccc}
\hline Distribution & \multicolumn{3}{c}{ Uniform } & \multicolumn{3}{c}{ Normal } \\
& 5 & 10 & 15 & 5 & 10 & 15 \\
\hline Machine & & & & & & \\
100 jobs & 1.11284 & 1.07673 & 1.04071 & 1.07082 & 1.05216 & 1.05210 \\
200 jobs & 1.10885 & 1.06516 & 1.02421 & 1.06953 & 1.04967 & 1.04013 \\
500 jobs & 1.10405 & 1.06016 & 1.01848 & 1.06874 & 1.04820 & 1.03421 \\
1000 jobs & 1.10193 & 1.05846 & 1.01507 & 1.06835 & 1.04715 & 1.02225 \\
1500 jobs 1.09988 & 1.05631 & 1.01291 & 1.06585 & 1.04639 & 1.01098 \\
\hline
\end{tabular}

TABLE 2: Results of $\mathrm{LB}(3) / \mathrm{LB}(2)$.

\begin{tabular}{ccccccc}
\hline Distribution & \multicolumn{3}{c}{ Uniform } & \multicolumn{3}{c}{ Normal } \\
& 5 & 10 & 15 & 5 & 10 & 15 \\
\hline Machine & & & & & & \\
100 jobs & 1.0632 & 1.0369 & 1.0362 & 1.0392 & 1.0310 & 1.0493 \\
200 jobs & 1.0313 & 1.0305 & 1.0271 & 1.0438 & 1.0321 & 1.0380 \\
500 jobs & 1.0453 & 1.0709 & 1.0254 & 1.0363 & 1.0248 & 1.0346 \\
1000 jobs & 1.0298 & 1.0456 & 1.0245 & 1.0340 & 1.0269 & 1.0259 \\
1500 jobs & 1.0199 & 1.0304 & 1.0220 & 1.0273 & 1.0309 & 1.0238 \\
\hline
\end{tabular}

from 1.04071 to 1.01291 when the number of jobs increases from 100 to 1500 . This phenomenon indicates the asymptotic optimality of the SPT rule. Contrarily, for the fixed number of jobs, ratios of SPT/LB(3) enlarge as the number of machines increases from 5 to 15 . The cause may be that the larger the number of machines, the larger the quantity of idle times inserted, which enlarges the gap between the value of objective and its lower bound.

The ratios $\mathrm{LB}(3) / \mathrm{LB}(2)$ showed in Table 2 are the values of $\mathrm{LB}(3)$ to $\mathrm{LB}(2)$. The data in the table reveal that $\mathrm{LB}(3)$ is obviously superior to $\mathrm{LB}(2)$ for moderate scale problems. As the number of jobs keeps enlarging, $\mathrm{LB}(3)$ approaches $\mathrm{LB}(2)$ more and that conforms the asymptotic optimality of $\mathrm{LB}(3)$.

\section{Conclusions}

In this paper, we investigate a very useful scheduling problem in steel production, the no-wait flow shop minimizing total completion time. The asymptotic optimality of the classical SPT rule is proven with the tool of asymptotic analysis when the problem scale is large enough. For the promotion of numerical simulation, a new lower bound with performance guarantee is given. Computational results show that the SPT rule and the new lower bound work well for large scale problems.

\section{Acknowledgments}

This research is partly supported by the National Natural Science Foundation of China (61104074), Fundamental Research Funds for the Central Universities (N110417005), China Postdoctoral Science Foundation (2013T60294, 20100471462), Scientific Research Foundation for Doctor of Liaoning Province of China (20101032), and the 2011 Plan Project of Shenyang City (F11-264-1-63).

\section{References}

[1] Y. N. Sotskov, T. Tautenhahn, and F. Werner, "Heuristics for permutation flow shop scheduling with batch setup times," $O R$ Spectrum, vol. 18, no. 2, pp. 67-80, 1996.

[2] Y. N. Sotskov, A. Allahverdi, and T.-C. Lai, "Flowshop scheduling problem to minimize total completion time with random and bounded processing times," Journal of the Operational Research Society, vol. 55, no. 3, pp. 277-286, 2004.

[3] R. L. Graham, E. L. Lawler, J. K. Lenstra, and A. H. G. R. Kan, "Optimization and approximation in deterministic sequencing and scheduling: a survey," Annals of Discrete Mathematics, vol. 5, pp. 287-326, 1979.

[4] H. Röck, "Some new results in flow shop scheduling," Zeitschrift für Operations Research A, vol. 28, no. 1, pp. 1-16, 1984.

[5] A. Allahverdi and T. Aldowaisan, "Minimizing total completion time in a no-wait flowshop with sequence-dependent additive changeover times," Journal of the Operational Research Society, vol. 52, no. 4, pp. 449-462, 2001.

[6] A. Allahverdi and T. Aldowaisan, "No-wait and separate setup three-machine flowshop with total completion time criterion," International Transactions in Operational Research, vol. 7, pp. 245-264, 2000.

[7] T. Aldowaisan and A. Allahverdi, "New heuristics for mmachine no-wait flowshop to minimize total completion time," Omega, vol. 32, no. 5, pp. 345-352, 2004.

[8] K. Gao, Q. Pan, P. N. Suganthan, and J. Li, "Effective heuristics for the no-wait flow shop scheduling problem with total flow time minimization," The International Journal of Advanced Manufacturing Technology, 66, pp. 1563-1572, 2013.

[9] A. Allahverdi and H. Aydilek, "Algorithms for no-wait flowshops with total completion time subject to makespan," The International Journal of Advanced Manufacturing Technology, vol. 68, no. 9, pp. 2237-2251, 2013.

[10] N. G. Hall and C. Sriskandarajah, "A survey of machine scheduling problems with blocking and no-wait in process," Operations Research, vol. 44, no. 3, pp. 510-525, 1996.

[11] D. Bai and T. Ren, "New approximation algorithms for flow shop total completion time problem," Engineering Optimization, vol. 45, no. 9, pp. 1091-1105, 2013. 


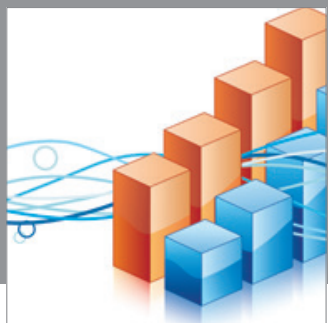

Advances in

Operations Research

mansans

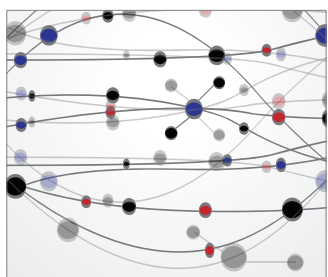

The Scientific World Journal
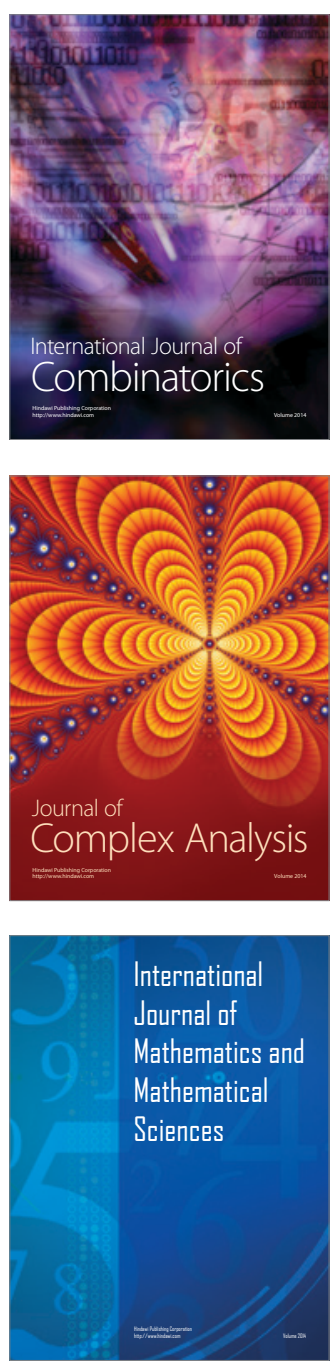
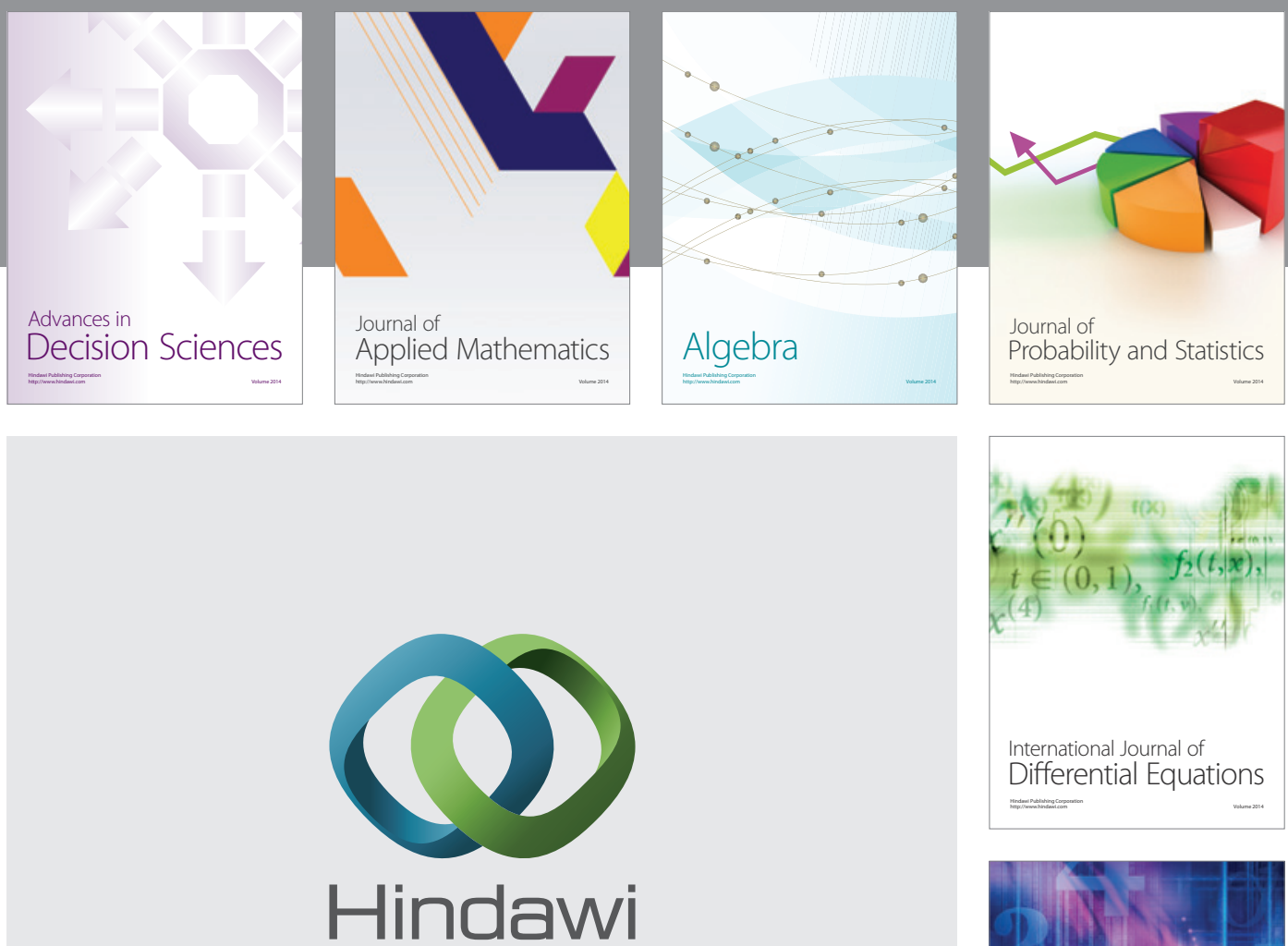

Submit your manuscripts at http://www.hindawi.com
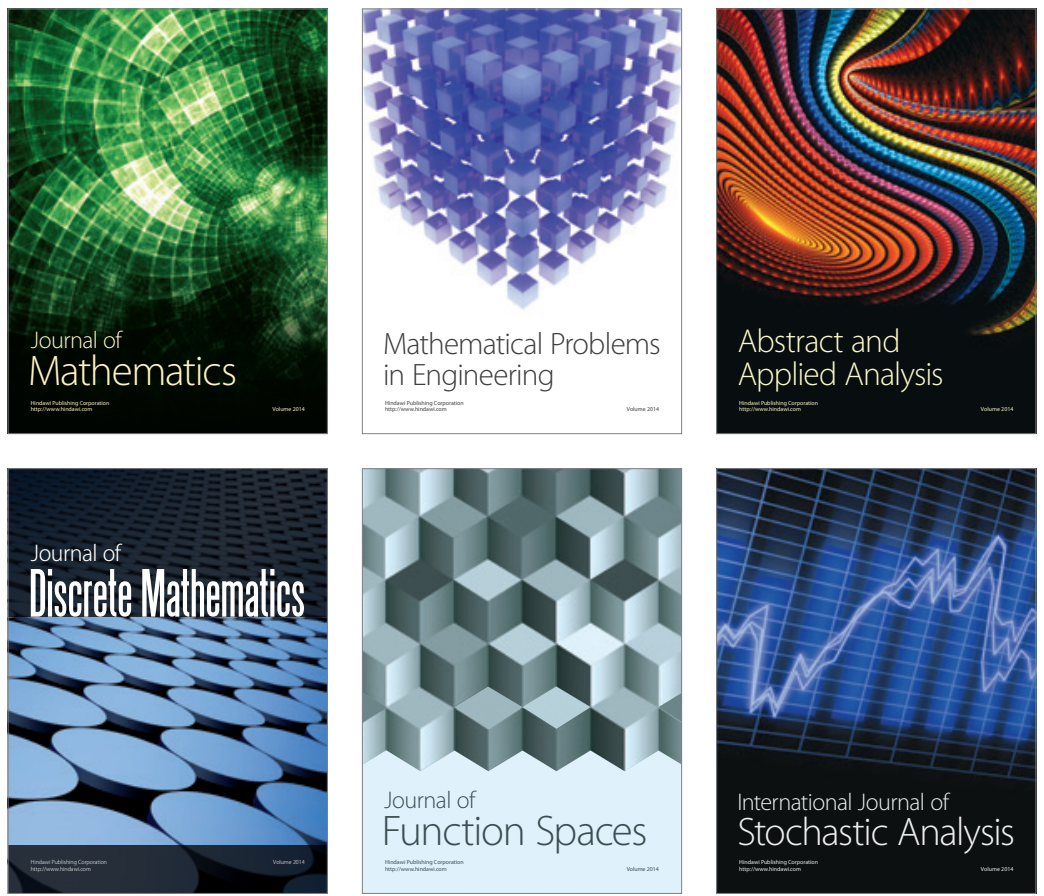

Journal of

Function Spaces

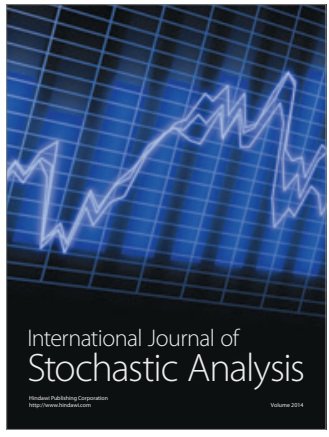

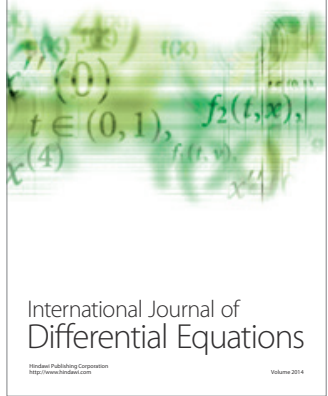
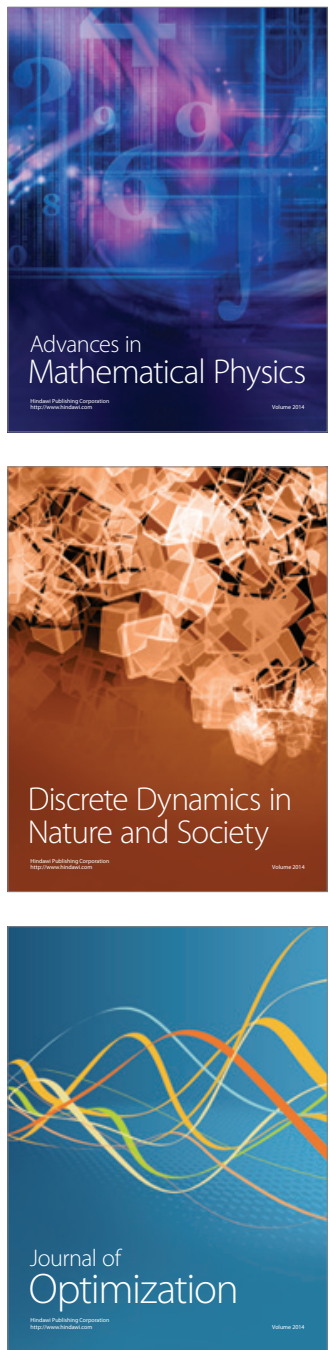